\title{
Processo de Avaliação do Programa Nacional de Melhoria do Acesso e da Qualidade da Atenção Básica: Revisão Integrativa
}

\author{
Milton Jorge Lobo Barbosa ${ }^{1}$, Pedro Paulo Rodrigues ${ }^{2}$, Aline Maria Lobo Barbosa ${ }^{3}$ \\ Luciano Moreira Alencar ${ }^{4}$, Evanira Rodrigues Maia ${ }^{5}$
}

\begin{abstract}
RESUMO
A Atenção Primaria à Saúde (APS) é definida como o primeiro nível de atenção à saúde da população do Sistema Único de Saúde (SUS), com a finalidade de oferecer à população uma atenção integral à saúde. Nesse contexto, o Ministério da Saúde criou, em 2011, o Programa Nacional de Melhoria do Acesso e da Qualidade da Atenção Básica (PMAQ), voltado para o estabelecimento de mudanças no processo de trabalho que resultem na qualidade e no acesso dos serviços, articulado com uma avaliação e uma certificação. Este artigo tem como objetivo analisar aspectos de efetivação do PMAQ-AB como sistema de avaliação profissional na APS. Trata-se de uma Revisão Integrativa realizada de março a julho de 2018. Teve-se como questão norteadora: "Quais os principais aspectos de avaliação da atenção profissional foram implantados pelo PMAQ-AB no Brasil?" Na seleção da amostragem foram incluídos os artigos originais e na íntegra disponíveis, publicados no idioma português, inglês e espanhol e que retratam a temática em questão. Os resultados mostraram que o processo de avaliação da atenção profissional faz parte desse programa, levando à busca pela melhoria na prestação de cuidados. No que se refere à condição de porta de entrada preferencial, os resultados apontam uma percepção das equipes da ESF em geral mais positiva que a dos usuários. Destaca-se que o estudo apresenta como limitações o número restrito de bases, sugerindo-se pesquisas mais amplas sobre a temática e que abordem minunciosamente os componentes da equipe e seu desempenho.
\end{abstract}

Palavras-chave: Programa Nacional de Melhoria do Acesso e da Qualidade da Atenção Básica. Atenção Primária à Saúde. Avaliação em Saúde.

\section{EVALUATION PROCESS OF THE NATIONAL PROGRAM TO IMPROVE ACCESS AND QUALITY OF BASIC ATTENTION:} INTEGRATIVE REVIEW

\section{ABSTRACT}

Primary Health Care (PHC) is defined as the first level of health care for the population of the Unified Health System (SUS), in order to provide the population with comprehensive health care. In this context, the Ministry of Health created in 2011 the National Program for Improving Access and Quality of Primary Care (PMAQ), aimed at establishing changes in the work process that result in quality and access to services, articulated with a evaluation and certification. This article aims to analyze aspects of PMAQ-AB implementation as a professional evaluation system in APS. This was an Integrative Review, carried out from March to July 2018. The guiding question was: "What were the main aspects of the assessment of professional care implemented by PMAQ-AB in Brazil?". In the selection of the sample, the original and full articles were published, published in Portuguese, English and Spanish, which portray the theme in question. The results showed that the process of evaluating professional attention is part of this program, leading to the search for improvement in care delivery. With regard to the preferential entry door condition, the results point to a perception of the ESF teams in general more positive than the users. It should be emphasized that the study presents as limitations the restricted number of bases, suggesting broader research on the subject and that minutily approach the components of the team and their performance.

Keywords: National Program for Improving Access and Quality of Primary Care. Primary Health Care. Health Assessment.

Recebido em: 9/8/2018

Aceito em: 25/10/2018

\footnotetext{
Secretaria de Saúde da Prefeitura de Caririaçu - CE. lobobarbosam@gmail.com

Enfermeiro especialista em Saúde Pública e Saúde da Família pela Faculdade Kurios, Maranguape, CE. pedro_roes@outlook.com

Cirurgiã-dentista na Secretaria Municipal de Saúde de Nova Olinda, CE. alineamlb@hotmail.com

4 Enfermeiro, mestrando em Cuidados Clínicos e Enfermagem pela Universidade Estadual do Ceará (Uece), Fortaleza, CE. luciano.m.alencar@gmail.com

Enfermeira, doutora em Enfermagem pela Universidade Federal do Ceará (UFC), Fortaleza, CE. evanira@bol.com.br
} 


\section{INTRODUÇÃO}

A Atenção Primaria à Saúde (APS) é definida como o primeiro nível de atenção à saúde da população no Sistema Único de Saúde (SUS), a qual se caracteriza como um conjunto de ações de saúde, sejam elas de modo individual e/ou coletivo, que busca a prevenção, promoção, reabilitação e manutenção da saúde, com a finalidade de oferecer à população uma atenção integral à saúde (SOSSAl et al., 2016).

A organização do trabalho na APS é essencial para que as equipes de Estratégia Saúde da Família (ESF) possam progredir na integração da atenção com a melhoria da assistência. Apesar disso, as ações das equipes ainda são planejadas de modo centralizado e pouco participativo. É importante que as ações de planejamento ampliem a visão crítica dos gestores, comunidade e profissionais da área, considerando que os processos autoavaliativos da saúde são usados para a reorganização do sistema, os quais vêm contribuindo significativamente para a melhoria da qualidade e do acesso na APS (MOREIRA; VIEIRA; COSTA, 2016).

Nesse contexto, o Ministério da Saúde criou, em 2011, o Programa Nacional de Melhoria do Acesso e da Qualidade da Atenção Básica (PMAQ-AB), por meio da Portaria no 1.654, um programa voltado para o estabelecimento de mudanças nos processos de trabalho que resultem na qualidade e no acesso aos serviços, articulando-os com uma avaliação e uma certificação (PINTO; SOUSA; FERLA, 2014; BRASIL, 2011).

O desenvolvimento do PMAQ depende da capacidade de mobilizar os seus usuários para buscarem mudanças nas condições e práticas da atenção, gestão e participação, e necessita da promoção de ambientes para diálogo, problematização, negociação e gestão de alterações entre as equipes, gestores e usuários, a fim de realizarem mudanças efetivas nos serviços de saúde (PINTO; SOUSA; FLORÊNCIO, 2012).

Esse programa encontra-se no terceiro ciclo de avaliação, estando divido em quatro fases: adesão e contratualização, desenvolvimento, avaliação externa e recontratualização. A cada ciclo novos parâmetros sobre a qualidade são determinados, os quais procuram a melhoria na qualidade das ações em saúde assim como a melhoria dos indicadores de saúde da população (GUIMARÃES; NERY, 2015).

$A$ avaliação Atenção Básica $(A B)$ é um método utilizado para identificar as fragilidades persistentes que dificultam a sua disposição e funcionamento no sentido da resolubilidade almejada para esse serviço (MICLOS; CALVO; COLUSSI, 2015).
Diante do exposto, torna-se evidente a necessidade de realizar estudos que possam analisar aspectos do PMAQ-AB como sistema de avaliação profissional na APS, como artifício para assegurar os princípios e diretrizes do SUS.

Ao analisar a importância do PMAQ na $A B$ na avaliação dos serviços do SUS, este estudo torna-se relevante na medida em que realça os diálogos sobre a avaliação da $A B$, no sentido de aproximar os trabaIhadores em saúde, gestores e usuários para a análise dos aspectos da integralidade da atenção. Ao mesmo tempo, colabora para a construção de uma reflexão e do entendimento sobre os conhecimentos dos profissionais de saúde acerca da avaliação na $A B$, como método que irá contribuir para o avanço dos serviços de saúde (ENGEL, 2015).

Este artigo tem como objetivo analisar aspectos de instituição do Programa Nacional de Melhoria do Acesso e da Qualidade da Atenção Básica como sistema de avaliação profissional na APS.

\section{MÉTODO}

Revisão Integrativa (RI) da literatura que foi realizada de março a junho de 2018, guiada por seis etapas: elaboração da questão da pesquisa, amostragem ou busca na literatura, categorização dos estudos, avaliação dos estudos incluídos na revisão, interpretação dos resultados e apresentação da revisão (MENDES; SILVEIRA; GALVÃO, 2008).

Para guiar o estudo, elaborou-se a questão norteadora: "Quais os principais aspectos de avaliação da atenção profissional foram implantados pelo Programa Nacional de Melhoria do Acesso e da Qualidade da Atenção Básica no Brasil?" Utilizou-se a estratégia Population, Variables and Outcomes (PVO) para definir palavra-chave e os Descritores em Ciências da Saúde (DeCS) adequados à pergunta de pesquisa, resultando em "Avaliação em Saúde" (outcomes), "Pessoal de saúde" (population) e "Atenção primária à saúde" (variables).

Quanto ao levantamento das produções na literatura, a busca foi realizada nas seguintes bases de dados: Literatura Latino-Americana e do Caribe em Ciências da Saúde (Lilacs); Cumulative Index to Nursing and Allied Heath Literature (CINAHL); Índice Bibliográfico Español en Ciencias de La Salud (Ibecs) e Medical Literature Analysis and Retrieval Sistem on-line (Medline). Em todas foi utilizado o método de busca avançada, quando foram utilizadas as palavras-chave "Programa Nacional de Melhoria do Acesso e da Qualidade da Atenção Básica" e os Descritores em 
Ciências da Saúde (DeCS) "Atenção primária à saúde". Para cruzamento desses termos foi empregado o operador booleano AND.

$\mathrm{Na}$ seleção da amostragem foram incluídos os artigos originais e na íntegra disponíveis, publicados nos idiomas português, inglês e espanhol e que retratassem a temática em questão. Definiu-se como recorte temporal de 2011 a 2018, considerando que o PMAQ foi lançado em 2011. Após a identificação foi realizada a seleção dos estudos segundo a questão norteadora e os critérios de inclusão delimitados, em que os estudos foram identificados por meio do método de busca e foram avaliados mediante a leitura na íntegra da publicação. Foi utilizado o fluxograma Prisma para demonstrar o processo de identificação, seleção, elegibilidade e inclusão dos estudos.

Para a caracterização dos estudos selecionados foi utilizado um checklist de elaboração própria contendo variáveis referentes a: autor(es); ano de publicação; título do artigo; objetivo; base de indexação/ periódico e principais resultados.

$\mathrm{Na}$ avaliação da qualidade dos estudos para composição da RI foi utilizada uma adaptação do Critical Appraisal Skills Programme (Casp) - Programa de habilidades em leitura crítica, integrante do Public Health Resource Unit (PHRU) (MOHER et al., 2009). Foram incluídas as evidências científicas classificadas de seis a dez pontos.

No processo de análise foi usado o método de redução de dados (WHITTEMORE; KNAFL, 2005). Os resultados foram apresentados a partir de quadros e em categorias temáticas e, posteriormente, discutidos com base na literatura atual pertinente. As categorias foram: o Programa Nacional de Melhoria do Acesso e da Qualidade da Atenção Básica e aspectos de avaliação da atenção profissional.

\section{RESULTADOS E DISCUSSÕES}

A análise foi composta por 16 estudos, sendo 15 publicados em Língua Portuguesa e 1 em Língua Inglesa, entre os anos de 2014 e 2017, a qual se iniciou com a identificação dos dados referentes aos autores, anos de publicação, título do artigo, periódico/base de dados, resultados, objetivos e pontuação Casp, conforme os Quadros 1 e 2 a seguir.

Figura 1 - Esquema dos trabalhos incluídos na amostra do estudo, segundo o Protocolo de Prisma

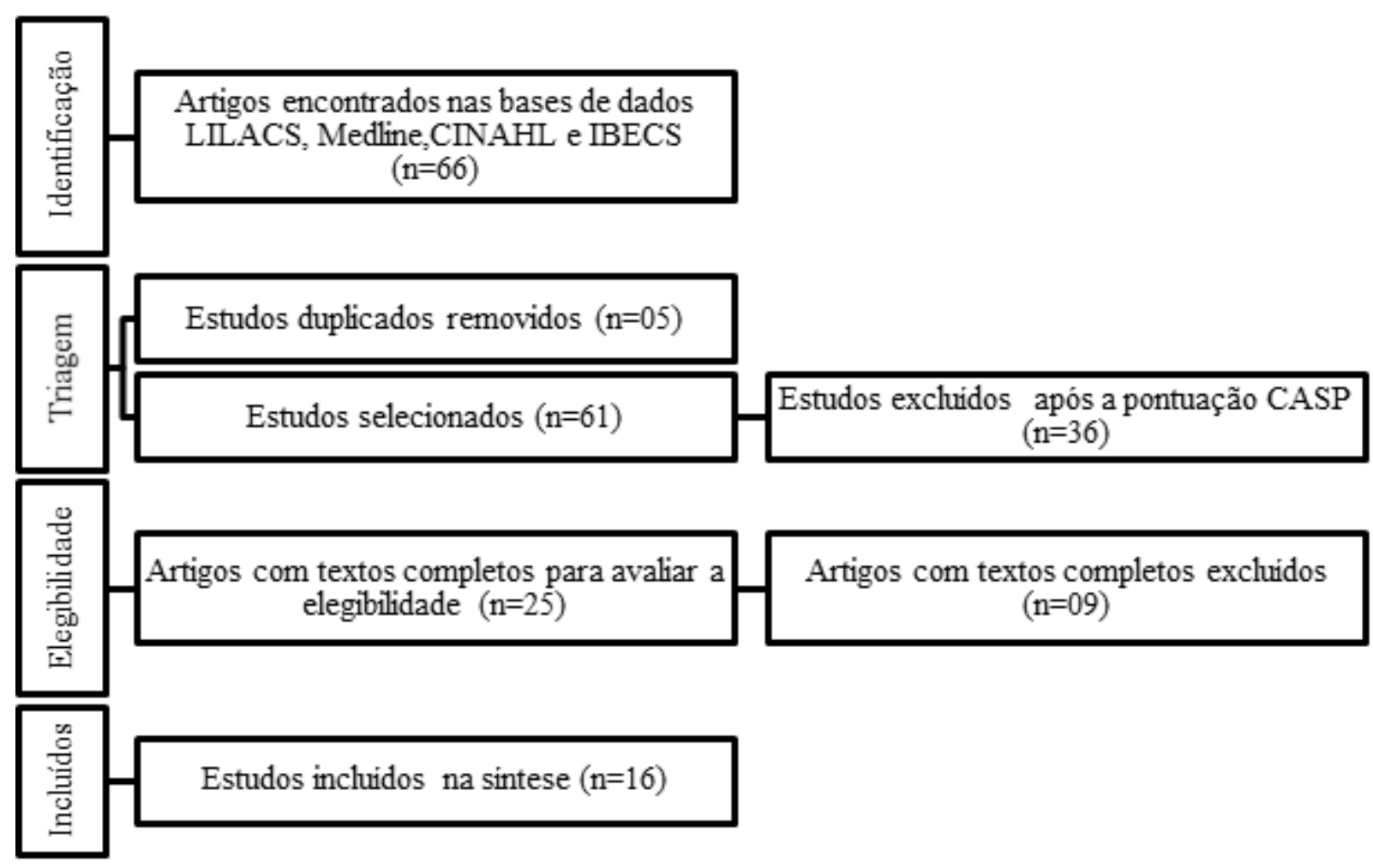

Fonte: MOHER et al., 2009. 
Processo de Avaliação do Programa Nacional de Melhoria do Acesso e da Qualidade da Atenção Básica:

Quadro 1 - Características dos estudos em relação aos dados de identificação. Crato, Ceará, Brasil, 2018

\begin{tabular}{|c|c|c|c|c|}
\hline $\begin{array}{l}\text { № DO } \\
\text { ESTUDO }\end{array}$ & AUTORES/ANO & TíTULO & PERIÓDICO/BASE & $\begin{array}{l}\text { PONTUA- } \\
\text { ÇÃO CASP }\end{array}$ \\
\hline 1 & $\begin{array}{l}\text { ALMEIDA; MARTIN; } \\
\text { CASOTTI, } \\
2017\end{array}$ & $\begin{array}{l}\text { Estratégias para consolidação da coordenação do } \\
\text { cuidado pela atenção à saúde. } \\
\text { Estratégia para consolidação da coordenação do } \\
\text { cuidado pela atenção à saúde. }\end{array}$ & $\begin{array}{l}\text { Revista Trabalho, Educação e Saúde } \\
\text { Lilacs }\end{array}$ & 6 \\
\hline 2 & $\begin{array}{l}\text { ALVES; ANDRADE; } \\
\text { SANTOS, } \\
2016\end{array}$ & $\begin{array}{l}\text { Longitudinalidade e formação profissional: funda- } \\
\text { mentos para o desempenho das equipes de saúde } \\
\text { da família }\end{array}$ & $\begin{array}{l}\text { Revista Saúde em Debate } \\
\text { Lilacs }\end{array}$ & 7 \\
\hline 3 & $\begin{array}{l}\text { BOUSQUAT et al., } \\
2017\end{array}$ & $\begin{array}{l}\text { Tipologia da estrutura das unidades básicas de } \\
\text { saúde brasileiras: os } 5 \text { Rs }\end{array}$ & $\begin{array}{l}\text { Cadernos de Saúde Pública } \\
\text { Lilacs }\end{array}$ & 6 \\
\hline 4 & FAUSTO et al., 2014 & $\begin{array}{l}\text { A posição da Estratégia Saúde da Família na rede } \\
\text { de atenção à saúde na perspectiva das equipes e } \\
\text { usuários participantes do } P M A Q-A B\end{array}$ & $\begin{array}{l}\text { Revista Saúde em Debate } \\
\text { Lilacs }\end{array}$ & 9 \\
\hline 5 & FEITOSA et al., 2016. & $\begin{array}{l}\text { Mudanças ofertadas pelo Programa Nacional de } \\
\text { Melhoria do Acesso e da Qualidade da Atenção } \\
\text { Básica }\end{array}$ & $\begin{array}{l}\text { Revista Saúde e Sociedade } \\
\text { Lilacs }\end{array}$ & 10 \\
\hline 6 & $\begin{array}{l}\text { FONTANA; LACERDA; } \\
\text { MACHADO, } \\
2016\end{array}$ & $\begin{array}{l}\text { O processo de trabalho na Atenção Básica à saúde: } \\
\text { avaliação da gestão }\end{array}$ & $\begin{array}{l}\text { Revista Saúde em Debate } \\
\text { Lilacs }\end{array}$ & 7 \\
\hline 7 & GARCIA et al., 2014 & $\begin{array}{l}\text { Análise da organização da Atenção Básica no Espí- } \\
\text { rito Santo: (des)velando cenários }\end{array}$ & $\begin{array}{l}\text { Revista Saúde em Debate } \\
\text { Lilacs }\end{array}$ & 7 \\
\hline 8 & JUNIOR; PINTO, 2014 & $\begin{array}{l}\text { Atenção Básica enquanto ordenadora da rede e } \\
\text { coordenadora do cuidado: Ainda uma utopia? }\end{array}$ & $\begin{array}{l}\text { Revista Divulgação em Saúde para } \\
\text { Debate } \\
\text { Lilacs }\end{array}$ & 8 \\
\hline 9 & $\begin{array}{l}\text { LIMÃO et al., } \\
2016\end{array}$ & $\begin{array}{l}\text { Equipamentos e insumos odontológicos e sua rela- } \\
\text { ção com as unidades da atenção primária à saúde }\end{array}$ & $\begin{array}{l}\text { Revista Brasileira de Promoção da } \\
\text { Saúde } \\
\text { Lilacs }\end{array}$ & 9 \\
\hline 10 & $\begin{array}{l}\text { MACHADO et al., } \\
2015\end{array}$ & $\begin{array}{l}\text { Programa saúde na escola: estratégia promotora } \\
\text { de saúde na atenção básica no Brasil }\end{array}$ & $\begin{array}{l}\text { Journal of Human Growth and De- } \\
\text { velopment } \\
\text { Lilacs }\end{array}$ & 6 \\
\hline 11 & $\begin{array}{l}\text { PINTO et al., } \\
2014\end{array}$ & $\begin{array}{l}\text { Atenção Básica e Educação Permanente em Saú- } \\
\text { de: cenário apontado pelo Programa Nacional de } \\
\text { Melhoria do Acesso e da Qualidade da Atenção } \\
\text { Básica (PMAQ-AB). }\end{array}$ & $\begin{array}{l}\text { Revista Divulgação em Saúde para } \\
\text { Debate } \\
\text { Lilacs }\end{array}$ & 6 \\
\hline 12 & $\begin{array}{l}\text { PINTO; SOUSA; FERLA } \\
2014\end{array}$ & $\begin{array}{l}\text { O Programa Nacional de Melhoria do Acesso e da } \\
\text { Qualidade da Atenção Básica: várias faces de uma } \\
\text { política inovadora }\end{array}$ & $\begin{array}{l}\text { Revista Saúde em Debate } \\
\text { LILACS }\end{array}$ & 9 \\
\hline 13 & $\begin{array}{l}\text { PROTASIO et al., } \\
2017\end{array}$ & $\begin{array}{l}\text { Satisfação do usuário da Atenção Básica em Saúde } \\
\text { por regiões do Brasil: 1o ciclo de avaliação externa } \\
\text { do PMAQ-AB }\end{array}$ & $\begin{array}{l}\text { Revista Ciência \& Saúde Coletiva } \\
\text { LILACS }\end{array}$ & 10 \\
\hline 14 & $\begin{array}{l}\text { RIBEIRO et al., } \\
2015\end{array}$ & $\begin{array}{l}\text { Acessibilidade aos serviços de saúde na Atenção } \\
\text { Básica do Estado de Goiás }\end{array}$ & $\begin{array}{l}\text { Revista Eletrônica de Enfermagem } \\
\text { LILACS }\end{array}$ & 6 \\
\hline 15 & $\begin{array}{l}\text { SOBRINHO et al., } \\
2015\end{array}$ & $\begin{array}{l}\text { Acesso e qualidade: avaliação das Equipes de Saú- } \\
\text { de Bucal participantes do PMAQ-AB } 2012 \text { em Per- } \\
\text { nambuco }\end{array}$ & $\begin{array}{l}\text { Revista Saúde em Debate } \\
\text { LILACS }\end{array}$ & 7 \\
\hline 16 & $\begin{array}{l}\text { TEIXEIRA et al., } \\
2014\end{array}$ & $\begin{array}{l}\text { Avaliação das práticas de promoção da saúde: um } \\
\text { olhar das equipes participantes do Programa Na- } \\
\text { cional de Melhoria do Acesso e da Qualidade \da } \\
\text { Atenção Básica }\end{array}$ & $\begin{array}{l}\text { Revista Saúde em Debate } \\
\text { LILACS }\end{array}$ & ---- \\
\hline
\end{tabular}

Fonte: Elaboração própria.

No que diz respeito ao período da publicação, os anos de 2008 e 2009 foram os que mais tiveram publicações pertinentes à temática, com $61,5 \%(n=8)$ e $23,1 \%(n=3)$, respectivamente. Foi possível verificar o decréscimo de publicações nos anos de 2010, 2011, 2012 e 2013. É oportuno assinalar que não foi encon- trada explicação para a diminuição no quantitativo de publicações em tais anos, posto que esta modalidade está cada dia mais presente no cenário do ensino dos cursos de saúde. É válido destacar que o ano de 2013 foi considerado apenas até o mês de julho. 
Quadro 2 - Características dos estudos em relação aos dados de identificação. Crato, Ceará, Brasil, 2018

\begin{tabular}{|c|c|c|}
\hline $\begin{array}{l}\text { № DO } \\
\text { ESTUDO }\end{array}$ & OBJETIVO & RESULTADOS \\
\hline 1 & $\begin{array}{l}\text { Analisar a coordenação do cuidado por meio } \\
\text { de dados do Programa Nacional para a Me- } \\
\text { Ihoria da Qualidade e do Acesso da Atenção } \\
\text { Básica. }\end{array}$ & $\begin{array}{l}\text { Os resultados indicaram que a atenção primária em saúde se consolidou como } \\
\text { porta de entrada preferencial. Os usuários relataram que as equipes de atenção } \\
\text { básica buscavam resolver seus problemas de saúde, o prontuário eletrônico es- } \\
\text { tava disponível, embora não fosse integrado aos demais níveis, e os profissionais } \\
\text { indicaram realizar reuniões semanais e receber apoio matricial. }\end{array}$ \\
\hline 2 & $\begin{array}{l}\text { Avaliar o desempenho de equipes de saúde } \\
\text { da família por meio do monitoramento dos } \\
\text { indicadores de saúde contratualizados no } \\
\text { PMAQ-AB. }\end{array}$ & $\begin{array}{l}\text { Os resultados revelam que as equipes com desempenho satisfatório em todos } \\
\text { os indicadores e em todos os anos analisados possuem maior tempo de estabe- } \\
\text { lecimento. }\end{array}$ \\
\hline 3 & $\begin{array}{l}\text { A tipologia da estrutura das } 38.812 \text { UBS } \\
\text { brasileiras foi elaborada com base nos resul- } \\
\text { tados do censo do ciclo } 1 \text { do PMAQ-AB. }\end{array}$ & $\begin{array}{l}\text { A tipologia aqui apresentada pode ser um instrumento para o acompanhamento } \\
\text { da qualidade da estrutura das UBS no país, temporal e espacialmente. }\end{array}$ \\
\hline 4 & \begin{tabular}{|l|} 
Analisar a posição da Estratégia Saúde da \\
Família na rede de atenção à saúde sob a \\
perspectiva das 16.566 equipes de Saúde da \\
Família e dos 62.505 usuários participantes \\
do PMAQ-AB em 2012 . \\
\end{tabular} & $\begin{array}{l}\text { Os resultados indicam que as equipes atuam cada vez mais como porta de entra- } \\
\text { da preferencial, atendendo a demandas diversas e exercendo a função de filtro } \\
\text { para a atenção especializada. }\end{array}$ \\
\hline 5 & $\begin{array}{l}\text { Analisar as mudanças que o PMAQ-AB têm } \\
\text { provocado nos serviços da atenção básica } \\
\text { em saúde. }\end{array}$ & $\begin{array}{l}\text { Foram apontadas três principais mudanças: organização do trabalho; recursos } \\
\text { materiais e infraestrutura da ESF; organização dos registros. }\end{array}$ \\
\hline 6 & $\begin{array}{l}\text { Desenvolver e aplicar um modelo de avalia- } \\
\text { ção do processo de trabalho na } A B \text { em saú- } \\
\text { de com foco na gestão municipal. } \\
\end{array}$ & $\begin{array}{l}\text { Existem grandes desafios para assegurar as condições necessárias à realização } \\
\text { dos aspectos processuais, em especial na continuidade do cuidado e na organi- } \\
\text { zação do trabalho. }\end{array}$ \\
\hline 7 & $\begin{array}{l}\text { Revelar aspectos da organização da } A B \text { à } \\
\text { Saúde nos } 78 \text { municípios do Estado do Espí- } \\
\text { rito Santo - Brasil -, no contexto avaliativo } \\
\text { do PMAQ-AB, no período de junho a setem- } \\
\text { bro de } 2012\end{array}$ & $\begin{array}{l}\text { Foram analisados três eixos: } 1 \text {. trabalho em saúde: evidenciou desprecarização } \\
\text { das relações de trabalho; } 2 \text {. processo de trabalho, planejamento e acolhimento: } \\
\text { dispositivos operados satisfatoriamente; } 3 \text {. estrutura, ambiência e acessibilida- } \\
\text { de: revelou dificuldades de utilização dos serviços pelos usuários em razão da } \\
\text { inadequação do ambiente. }\end{array}$ \\
\hline 8 & $\begin{array}{l}\text { Identificar políticas do próprio MS que bus- } \\
\text { cam fortalecer a AB nestes papéis, fazendo } \\
\text { uma busca especial entre aquele conjunto } \\
\text { de ações que, internacionalmente, são reco- } \\
\text { nhecidas como pró-coordenação. }\end{array}$ & $\begin{array}{l}\text { Identificou políticas do próprio MS que buscam fortalecer a AB nestes papéis, } \\
\text { fazendo uma busca especial entre aquele conjunto de ações que, internacional- } \\
\text { mente, são reconhecidas como pró-coordenação. }\end{array}$ \\
\hline 9 & $\begin{array}{l}\text { Analisar a relação entre equipamentos/ } \\
\text { insumos odontológicos e as unidades de } \\
\text { atenção primária à saúde. }\end{array}$ & $\begin{array}{l}\text { Encontrou-se um número reduzido de equipamentos e insumos odontológicos, } \\
\text { como autoclaves em condições de uso em apenas } 27,2 \% \text { dos postos de saúde e } \\
\text { em } 29,0 \% \text { das UBS, e selantes em quantidade suficiente apenas em } 31,7 \% \text { dos } \\
\text { postos de saúde e em } 30,7 \% \text { das UBS. }\end{array}$ \\
\hline 10 & $\begin{array}{l}\text { Identificar e descrever as ações desenvolvi- } \\
\text { das pelas ESF no Programa Saúde na Escola } \\
\text { (PSE), participantes do PMAQ-AB. } \\
\end{array}$ & $\begin{array}{l}\text { Os resultados mostraram que alguns itens, como a capacitação dos profissionais } \\
\text { para trabalhar com educação e saúde, precisam ser fortalecidos. }\end{array}$ \\
\hline 11 & $\begin{array}{l}\text { Analisar questões relativas ao ensino/apren- } \\
\text { dizagem no cotidiano da } A B \text {, conforme os } \\
\text { dados coletados na etapa de avaliação ex- } \\
\text { terna do PMAQ-AB }\end{array}$ & $\begin{array}{l}\text { A EPS teve expansão e abrangência importantes na AB; que as equipes têm se } \\
\text { conectado com ofertas formativas; que ainda há evidência de processos educa- } \\
\text { tivos pontuais e de cunho informacional; e que as variações regionais de acesso } \\
\text { às tecnologias de informação e comunicação são produzidas pelos problemas de } \\
\text { infraestrutura, não pelo interesse e adesão. }\end{array}$ \\
\hline 12 & $\begin{array}{l}\text { Analisar os diferentes papéis do programa } \\
\text { na nova Política Nacional de Atenção Básica. }\end{array}$ & $\begin{array}{l}\text { Permitiu perceber um incremento nos recursos destinados, no âmbito federal, à } \\
\text { AB e um percurso inovador no que se refere ao conteúdo da política, à mobiliza- } \\
\text { ção local e à produção de uma cultura de monitoramento e avaliação embasada } \\
\text { na utilização de indicadores de autoavaliação e de Avaliação Externa. }\end{array}$ \\
\hline 13 & $\begin{array}{l}\text { Identificar os principais fatores que influen- } \\
\text { ciam na Satisfação do Usuário com os servi- } \\
\text { ços de Atenção Básica por regiões do Brasil. }\end{array}$ & $\begin{array}{l}\text { Os fatores influenciadores da satisfação dos usuários da AB variam de acordo } \\
\text { com a região e estão relacionados principalmente à qualificação do acesso, ao } \\
\text { atendimento das necessidades dos usuários e aos aspectos do processo de tra- } \\
\text { balho. }\end{array}$ \\
\hline 14 & $\begin{array}{l}\text { Analisar a acessibilidade aos serviços de } \\
\text { saúde na } A B \text { no Estado de Goiás. }\end{array}$ & $\begin{array}{l}\text { Verificou-se que a sala de acolhimento está ausente em } 68,5 \% \text { das unidades } \\
\text { de saúde, o que prejudica, consideravelmente, os profissionais nas tomadas de } \\
\text { decisão imediatas. A ausência de consultórios clínicos em } 2 \% \text { dos locais dificulta } \\
\text { a acessibilidade aos serviços de saúde na Atenção Básica em Goiás. }\end{array}$ \\
\hline 15 & $\begin{array}{l}\text { Avaliar o acesso e a qualidade das ações e } \\
\text { serviços das EqSB participantes do PMAQ-AB } \\
\text { no Estado de Pernambuco no ano de } 2012 \text {. } \\
\end{array}$ & $\begin{array}{l}\text { Foi constatada disponibilidade de equipamentos e insumos odontológicos garan- } \\
\text { tindo a realização de procedimentos clínico-cirúrgicos, com exceção dos referen- } \\
\text { tes à prótese dentária. }\end{array}$ \\
\hline 16 & $\begin{array}{l}\text { Sistematizar e analisar as práticas de PS } \\
\text { desenvolvidas pelas equipes de Atenção } \\
\text { Básica (EqAB) que participaram da etapa de } \\
\text { avaliação externa do Programa Nacional de } \\
\text { Melhoria de Acesso e da Qualidade da Aten- } \\
\text { ção Básica - PMAQ-AB. }\end{array}$ & $\begin{array}{l}\text { Conclui-se que as práticas são fragmentadas e focalizadas; urge integrar, equili- } \\
\text { bradamente, cuidado clínico, prevenção e promoção da saúde. }\end{array}$ \\
\hline
\end{tabular}


O programa nacional de melhoria do acesso e da qualidade da atenção básica

O PMAQ-AB teve o início do seu primeiro ciclo em 2012 como parte da nova Política Nacional de Promoção da Saúde (BRASIL, 2014), que fez revisão da Portaria № 687 de 30 de março de 2006 e, mais recentemente, foi alterada pela Portaria № 1645 de outubro de 2015, que dispõe sobre as regras do PMAQ para a $A B$ (incluindo saúde bucal e Nasf) com o propósito de modificar as condições de infraestrutura e de funcionamento das Unidades Básicas de Saúde da Família (UBSF), melhorar o acesso dos usuários aos serviços e prover uma melhor atenção, cuidado e promoção de saúde na Atenção Básica (AB), por meio do desenvolvimento de processos contínuos de avaliação e de incentivos financeiros às equipes certificadas (ALMEIDA; MARTIN; CASOTTI, 2017).

Esse programa almeja ampliar a capacidade das três esferas de governo na disponibilidade de serviços que garantam um padrão de qualidade comparável nos âmbitos nacional, regional e local (BOUSQUAT et al., 2017), de modo que permita uma maior transparência e efetividade das ações governamentais direcionadas à $A B$ em todo o Brasil (RIBEIRO et al., 2015; GARCIA et al., 2014, p. 5).

$O P M A Q-A B$ entrou em cena como um programa estruturado para remodelar aspectos essenciais da $A B$. Visando a atingir vários níveis dentro das UBS, o programa em questão surgiu como uma possibilidade de efetividade em todas as esferas, bem como meIhoria das condições do cuidado em saúde que perpassam a assistência e seus microprocessos.

O PMAQ-AB está disposto em quatro fases inter-relacionadas, as quais, juntas, formam um ciclo contínuo, buscando melhorias no acesso e na qualidade mediante a adesão e a contratualização das equipes, a pactuação de compromissos nas três esferas de gestão no desenvolvimento das ações de monitoramento dos indicadores de saúde, no estabelecimento de processos autoavaliativos, nas estratégias de educação permanente, na avaliação externa e na recontratualização (ALVES; ANDRADE; SANTOS, 2016; MOTA; DAVID, 2014).

Por meio da avaliação da qualidade dos serviços na ESF, o PMAQ-AB procura verificar a efetividade da incorporação dos princípios do SUS. Quanto à integralidade, a qual é apontada como sendo o conjunto de ações e de serviços que garante a assistência em saúde nos distintos níveis de atenção e a longitudinalidade, que garante continuação dos serviços odontológicos no tempo, é frequentemente rompida quando há indisponibilidade de insumos/materiais e equipamentos para a oferta de atenção ao usuário (LIMÃO et al., 2016).

A consolidação do SUS pode ser repensada conforme os dados advindos do PMAQ-AB e acompanhada pela sociedade no portal do Departamento da Atenção Básica, principalmente verificando se há mecanismos que assegurem a comparação entre as ações dos diversos serviços de saúde nas várias regiões do país, assim como se está sendo incremental por meio de um processo contínuo de melhoramento de padrões e indicadores de acesso e qualidade, e se está havendo transparência e envolvimento de todos os atores sociais envolvidos. Todo esse ciclo visa a resguardar os princípios e diretrizes do sistema em tela, e essa análise contribui para uma maior aproximação com as realidades locais.

Entre os objetivos da $A B$, que são pactuados no $P M A Q-A B$, merecem destaque as ações voltadas para a saúde da mulher, quando os indicadores de desempenho definidos estão em concordância com a Rede Cegonha, a qual foi desenvolvida com o propósito de organizar a assistência por intermédio de uma rede de cuidados que garante o direito sexual e reprodutivo às mulheres. (ALVES; ANDRADE; SANTOS, 2016).

Apesar de ser um programa recém-criado, o $P M A Q-A B$ vem sendo questionado nos aspectos que respeitam aos processos de trabalho nas equipes (MACHADO et al., 2015). Os aspectos que estimulam a relação competitiva entre equipes de saúde e a obtenção do reconhecimento por mérito, basicamente associada com os indicadores de resultados, tendo como ênfase a redução de doenças, levam a questionar em que ponto não está progredindo na obtenção de uma saúde por resultados, em lugar de reafirmar o direito universal à saúde integral (MOTA; DAVID, 2014).

No processo de avaliação as equipes, nas UBS, acabam por buscar o alcance de metas. Indicadores são trabalhados intensamente no intuito de que o dispositivo seja considerado dentro dos padrões estabelecidos. Por vezes, essa busca estimula a competição entre as unidades e suas equipes.

Na educação permanente em saúde o PMAQ-AB possui aspectos de investigação e é uma de suas principais apostas, tanto para a produção de movimentos locais quanto na singularização, suporte, qualificação e reinvenção desse movimento de mudança nas práticas de atenção, gestão, educação e participação (PINTO et al., 2014). 
Entre os indicadores investigados pela Avaliação Externa do PMAQ estão "a visita domiciliar e os cuidados realizados no domicílio. A primeira é destacada como um indicador de mudança do modelo de atenção, alinhada aos princípios da $A B$, como a integralidade e a continuidade do cuidado, o acesso, a eqüidade, a humanização e o vínculo" (DE-CARLI et al., 2015, p. 3).

A abordagem à educação permanente e o destaque à visita domiciliar apontam como o PMAQ-AB possui uma estrutura diferenciada, que busca fortalecer os processos formativos e a integralidade do cuidado, rompendo as ligações com processos mais rígidos e verticalizados, quando a assistência se dá de maneira objetiva e limitada.

Outro ponto que faz parte do PMAQ-AB é a Avaliação Externa, a qual foi criada com a função de avaliar as condições de acesso e a qualidade dos serviços de saúde para os municípios e para as equipes de profissionais de saúde cadastradas no programa por meio do monitoramento dos indicadores contratualizados e pela investigação dos padrões de qualidade das equipes (PROTASIO et al., 2017).

A referida avaliação permite conhecer a realidade das unidades. Trata-se de uma etapa fundamental para ter contato com as equipes e o atendimento prestado. Na avaliação externa reduz as possibilidades de alteração de dados, de modo que as visitas se dão nas unidades, coletando dados diretamente do que se está observando e constando.

\section{Aspectos de avaliação da atenção profissional}

O PMAQ-AB foi criado como sendo uma estratégia da PNAB, voltada para a mobilização dos atores sociais locais para enfrentar problemas e situações entendidas como condicionantes do desenvolvimento e da qualificação da AB no Brasil (PINTO; SOUSA; FERLA, 2014).

As equipes de profissionais de saúde devem assumir o compromisso de seguir as regras do programa e as diretrizes organizacionais do processo de trabalho, as quais incluem: a introdução de padrões no acesso e na qualidade da gestão colegiada, no planejamento e na avaliação; ser monitorada por seis meses a partir dos indicadores pactuados; e, finalmente, passar por um processo de avaliação que inclui a realização da autoavaliação, o desempenho nos indicadores monitorados e a avaliação in loco, realizada por avaliadores do programa (PINTO; SOUSA; FERLA, 2014).
O processo de avaliação da atenção profissional pauta-se no compromisso dos profissionais da $A B$ em atuarem com base nas diretrizes do MS, respeitando as particularidades locais e exercendo suas funções com a priorização da promoção e prevenção da saúde, bem como ofertando um atendimento de qualidade e eficaz.

No campo da produção do cuidado em odontologia o trabalho dos profissionais da equipe de saúde bucal (ESB) deve ser centralizado no combate aos principais agravos à saúde bucal dos pacientes, aliado às estratégias que assegurem maior acesso ao cuidado em ciclos incrementais de melhoria da resolutividade do serviço ofertado (SOBRINHO et al., 2015).

No estudo desenvolvido por Pimentel et al. (2010), identificaram-se as dificuldades no envolvimento de procedimentos coletivos voltados para a promoção e a prevenção em saúde bucal, quando se observam os esforços para superar o modelo de atenção para o desenvolvimento de práticas curativas. Esses achados mostram que ainda não existe uma adesão efetiva pelas equipes no que diz respeito à Política Nacional de Saúde Bucal no que se refere à ampliação e qualificação da AB (SOBRINHO et al., 2015).

Dessa forma, ainda persistem deficiências na coordenação do cuidado e também na gestão clínica de casos que necessitam de cuidado continuado. A estruturação do serviço possui como desafio o enfrentamento dos "vazios assistenciais" nos serviços de média complexidade em saúde bucal (SOBRINHO et al., 2015). Ainda existem deficiências na coordenação do cuidado e também na gestão clínica de casos que necessitam de cuidado continuado. Embora essas ações sejam, há muito tempo, pauta dos processos formativos e também de protocolos, o que percebemos é que há uma diferença importante entre "o que deveria ser" e "o como ocorre de fato" (JUNIOR; PINTO, 2014).

A valorização da existência de uma equipe de saúde bucal está na sua importância nas políticas de saúde dos últimos anos e na execução dos programas específicos da saúde bucal (Brasil Sorridente), instalando Centros de Especialidades Odontológicas (Ceos) municipais e regionais, visando à garantia da integralidade da atenção. A demora no oferecimento de serviços de maior complexidade em saúde bucal, contudo, pode originar sérios problemas de saúde, os quais podem ocasionar um profundo impacto na qualidade de vida e da saúde dos pacientes em algumas regiões do país. "A estratégia brasileira de disponibilizar o acesso ao cuidado oral no mesmo espaço das demais práti- 
cas de saúde de atenção primária à saúde, ou seja, em UBS, tem sido reconhecida em outras realidades como bastante positiva" (BOUSQUAT et al., 2017, p. 5).

Diversas são as causas atribuídas ao não cumprimento das recomendações do MS, de modo que muitas equipes ainda possuem dificuldades em serem guiadas pelas mesmas. Como resultados, há falhas significativas na atuação da $A B$, desenvolvendo fragilidades ao longo dos contatos com a comunidade, podendo esses aspectos serem avaliados pelo $\mathrm{PMAQ}-\mathrm{AB}$.

O comprometimento dos cuidados prestados advém não somente da dificuldade de fixação dos profissionais à comunidade. Fatores como a falta de insumos, maquinário e instrumentais, as condições de trabalho inadequadas e a precarização da estabilidade profissional influenciam negativamente no alcance de metas, o que leva a uma avaliação, por vezes, negativa de muitos profissionais e equipes.

A rotatividade dos profissionais de saúde compromete o estabelecimento de vínculo com a população, criando, desse modo, obstáculos no estabelecimento dos pilares da integralidade nas ações, as quais influenciam na qualidade da assistência (ALVES; ANDRADE; SANTOS, 2016).

Sobre a rotina do atendimento desenvolvida pelos profissionais de saúde, ainda se observam dificuldades voltadas para a sua efetivação, levando em consideração as diversas complexidades das demandas das comunidades que necessitam de um olhar diferenciado (MOTA; DAVID, 2014). Para isso, as equipes de saúde podem, via PSE, realizar ações de saúde em conjunto com as escolas, uma vez que essas apresentam um bom índice de desenvolvimento de atividades coletivas (MACHADO et al., 2015).

Os dados expostos pelo PMAQ-AB nas ações de Educação Permanente na área da saúde, mostram que as políticas do SUS se estabeleceram de maneira expressiva na $A B$ em oito anos de existência. Os esforços na análise do PMAQ-AB podem ser uma contribuição para a procura de um maior potencial de modificação (PINTO et al., 2014).

Ao se realizar a avaliação da atenção profissional constata-se que a práxis não se efetiva em algumas situações. O que é preconizado pelo MS não ocorre no âmbito das unidades, gerando discussões a respeito das reais possibilidades que as gestões ofertam às equipes, bem como os desafios encontrados e as perspectivas de melhoria.

O PMAQ-AB possibilita o desenvolvimento de mudanças, além de induzir e estimular as modificações estruturais e a avaliação e monitoramento do desempenho permanente da AB. Ainda considera-se, entretanto, o espaço físico inapropriado, e a falta de equipamentos profissionais influencia a qualidade da assistência à população (FEITOSA et al., 2016).

Ao se avaliar as condições de trabalho é possível associar as práticas ali exercidas as suas possibilidades. A avaliação ampla permite que os gestores, bem como os demais interessados, percebam que refletir sobre precarização das relações de trabalho e vínculo empregatício faz-se importante, considerando que esta pode ser um dos motivos para a insatisfação no ambiente de trabalho e seus consequentes déficits.

Outra mudança e contribuição que foi adquirida após a consolidação do PMAQ-AB no município de Grossas - RN -, foi a organização dos registros, a qual foi mencionada pelos profissionais de saúde e gestores como melhoria do atendimento da ESF, uma vez que racionaliza melhor o acesso e, por consequência, garante um menor tempo de espera (FEITOSA et al., 2016).

No que se refere aos aspectos estruturais, foi identificado que não asseguram o desenvolvimento de um processo de trabalho com qualidade na $A B$. "Os profissionais e gestores municipais de saúde precisam retomar o entendimento do processo de trabaIho como um objeto contínuo do trabalho em equipe. Atualmente, ele é executado por meio de instrumentos fragmentados, que diminuem a qualidade dos serviços e comprometem o potencial de resolubilidade da AB" (FONTANA; LACERDA; MACHADO, 2016, p. 7).

Os resultados do PMAQ-AB devem ser amplamente utilizados em prol da melhoria da $A B$, reconhecendo suas fragilidades e fortalezas, investindo nos aspectos que estão em falta, trabalhando sobre todos os itens que necessitam de um novo olhar, uma nova roupagem. Os dados devem servir como uma bússola em busca dos resultados almejados.

Uma estrutura física comprometida ou inadequada pode inviabilizar a efetivação da integralidade, princípio esse assegurado na criação do SUS. Esse princípio implica o desenvolvimento de ações e serviços voltados para a garantia da promoção, proteção e reabilitação, e é compreensível que a estrutura física interfira de forma direta na continuidade do cuidado à saúde (LIMÃO et al., 2016).

A inexistência de uma estrutura adequada pelas UBS prejudica o atendimento, pois os profissionais ficam desprovidos de um local adequado para priorizar a assistência à saúde de acordo com as necessidades individuais e prioritárias de cada indivíduo, originando demora ou atendimento inadequado (RIBEIRO et al., 2015). 
Frequentemente as UBSF apresentam problemas de infraestrutura pela falta de investimento em manutenção/ampliação, o que leva os profissionais dessas unidades a culpabilizarem a estrutura predial pelo não cumprimento de metas do programa. Essas discussões somente vêm se ampliando à medida que os investimentos em saúde são reduzidos rotineiramente. Isso deixa de ser um problema específico da atenção profissional, passando à reflexão sobre a gestão local da AB.

Outro ponto avaliado no PMAQ-AB foi a precariedade dos recursos humanos nos municípios, que, apesar de nenhum apoio técnico especializado, alta rotatividade de profissionais e sobrecarga de trabaIho, vem ampliando as estratégias de modo eficaz, embora ainda fragilizada. Já nos recursos tecnológicos evidencia-se que o Ministério da Saúde mostrou problemas na obtenção de equipamentos para os avaliadores externos. Nos municípios essa dificuldade se agrava, tendo em vista que o programa prevê um sistema de informações na rede. A realidade existente é de equipamentos de informática obsoletos, ausência de internet ou mesmo internet lenta e dificuldades de técnicos de informática (LINHARES; LIRA; ALBUQUERQUE, 2014).

Diversas são as dificuldades na ampliação das estratégias intersetoriais para além do setor saúde, as quais se justificam pela dificuldade de os profissionais da $A B$ articularem o planejamento e execução de ações integradas aos demais setores sociais (TEIXEIRA et al., 2014).

Em relação à formação e qualificação dos profissionais, sejam eles médicos, enfermeiros e cirurgiões-dentistas, é evidente a necessidade de oferta de especializações e residências para melhor capacitar os recursos humanos na $A B$ (SEIDL et al., 2014).

\section{CONSIDERAÇÕES FINAIS}

Entre os programas voltados para a ampliação do acesso e a melhoria da qualidade dos serviços e programas de saúde, destaca-se o PMAQ-AB, o qual procura garantir que este nível de atenção se configure como sendo a porta de entrada para os serviços de saúde. $O$ processo de avaliação da atenção profissional faz parte desse programa, levando à busca pela melhoria na prestação de cuidados.

Em relação à condição de porta de entrada preferencial, os resultados apontam uma percepção das equipes da ESF, em geral, mais positiva que a dos usuários. Pela visão dos profissionais, as UBS apresentam a condição de uma porta aberta, com destaque para alguns componentes do funcionamento: organização da agenda, atendimento da demanda espontânea e acesso às consultas de $A B$. Por outro lado, as respostas dos usuários, "consumidores" diretos desses serviços de saúde, apontam a importância de realizar avanços quanto à organização funcional, mais visíveis no que respeita ao acolhimento, à disponibilidade da UBS para consultas de urgência e às formas de acesso às consultas.

Destaca-se que este estudo apresenta como limitações o número restrito de bases, sugerindo-se pesquisas mais amplas sobre a temática e que abordem, mais detidamente, os componentes da equipe e seu desempenho.

\section{REFERÊNCIAS}

ALMEIDA, P. F.; MARTIN, J.; CASOTTI, E. Estratégias para consolidação da coordenação do cuidado pela atenção básica. Revista Trabalho, Educação e Saúde, Rio de Janeiro. v. 15, n. 2, maio/ago. 2017. Disponível em: <www.scielo.br/scielo. php?script=sci_arttext\&pid=S1981-77462017000200373 $>$. Acesso em: 20 jun. 2018.

ALVES, C. R.; ANDRADE, M. C.; SANTOS, C. L. S. Longitudinalidade e formação profissional: fundamentos para o desempenho das equipes de saúde da família. Revista Saúde em Debate, v. 40, n. 111, out./dez. 2016. Disponível em: <https://www.scielosp.org/scielo.php?script=sci_arttext\&pi$d=$ S0103-11042016000400268>. Acesso em: 20 jun. 2018.

BOUSQUAT, A. et al. Tipologia da estrutura das unidades básicas de saúde brasileiras: os 5 R. Caderno de Saúde Pública, Rio de Janeiro. v. 33, n. 8, ago. 2017. Disponívelem: $<w w w . s c i e l o . b r / s c i e l o . p h p ? s c r i p t=s c i \_a r t t e x t \&$ pid =S0102=311-2017000805005X\&lng=pt\&nrm=iso\&tlngpt>. Acesso em: 20 jun. 2018.

BRASIL. Ministério da Saúde. Portaria GM no 1.654 de 19 de julho 2011. Institui, no âmbito do Sistema Único de Saúde, o Programa Nacional de Melhoria do Acesso e da Qualidade da Atenção Básica (PMAQ-AB) e o Incentivo Financeiro do PMAQ-AB, denominado Componente de Qualidade do Piso de Atenção Básica Variável - PAB Variável. Diário Oficial da União, Brasília, DF, 2011. Disponível em: <http://bvsms.saude.gov.br/bvs/saudelegis/gm/2011/prt1654_19_07_2011. html>. Acesso em: 20 fev. 2018.

Ministério da Saúde. Política Nacional de Promoção da Saúde. Revisão da portaria MS/GM no 687 de 30 de março de 2006. Brasília, DF, 2014. Disponível em: <http://bvsms.saude.gov.br/bvs/saudelegis/gm/2011/ prt1654_19_07_2011.html . Acesso em: 20 fev. 2018.

. Ministério da Saúde. Secretaria de Atenção à Saúde. Departamento de Atenção Básica. Programa Nacional de Melhoria do Acesso e da Qualidade da Atenção Básica (PMAQ-AB): manual instrutivo. Departamento de Atenção Básica. Brasília: Ministério da Saúde, 2017. Disponível em: <http://bvsms.saude.gov.br/bvs/saudelegis/gm/2011/ prt1654_19_07_2011.html>. Acesso em: 20 fev. 2018. 
CRITICAL APPRAISAL SKILLS PROGRAMME (Casp). Great Ormond Street Hospital for Children. mar. 2011. Disponível em: <http://www.ucl.ac.uk/ich/services/library/training_ material/critical-appraisal>. Acesso em: 25 maio 2018.

DE-CARLI, A. D. et al. Visita domiciliar e cuidado domiciliar na Atenção Básica: um olhar sobre a saúde bucal. Revista Saúde em Debate, Rio de Janeiro. v. 39, n. 105, abr./jun. 2015. Disponível em: <www.scielo.br/scielo.php?script=sci_arttext\&pid=S0103-11042015000200441>. Acesso em: 20 jun. 2018.

ENGEL, R. H. Avaliação do PMAQ sob a ótica dos gestores municipais de saúde no interior do Rio Grande do Sul. 90 f. 2015. Dissertação (Mestrado) - Universidade Federal de Santa Maria, Santa Maria, 2015. Disponível em: <coral. ufsm.br/ppgenf/images/Dissertacoes_alunos/Dissertacao_ Rosana_Huppes_Engel.pdf>. Acesso em: 3 jun. 2018.

FAUSTO, M. C. R. et al. A posição da Estratégia Saúde da Família na Rede de Atenção à Saúde na perspectiva das equipes e usuários participantes do PMAQ-AB. Revista Saúde em Debate, Rio de Janeiro. v. 38, out. 2014. Disponível em: <www.scielo.br/scielo.php?script=sci_arttext\&pi$d=$ 00103-11042014000600013 >. Acesso em: 20 jun. 2018.

FEITOSA, R. M. M. et al. Mudanças ofertadas pelo Programa Nacional de Melhoria do Acesso e da Qualidade da Atenção Básica. Revista Saúde e Sociedade, São Paulo, v. 25, n. 3, jul./set. 2016. Disponível em: <www.scielo.br/scielo.php?script=sci_arttext\&pid=S0104-12902016000300821>. Acesso em: $2 \overline{0}$ jun. 2018.

FONTANA, K. C.; LACERDA, J. T.; MACHADO, P. M. O. O processo de trabalho na Atenção Básica à Saúde: avaliação da gestão. Revista Saúde em Debate, Rio de Janeiro, v. 40, n. 110, jul./set. 2016. Disponível em: <www.scielo.br/scielo. php?script=sci_arttext\&pid=S0103-11042016000300064>. Acesso em: 20 jun. 2018.

GARCIA, A. C. P. et al. Análise da organização da Atenção Básica no Espírito Santo: (des)velando cenários. Revista Saúde em Debate, Rio de Janeiro. v. 38, out. 2014. Disponível em: <www.scielo.br/scielo.php?script=sci_arttext\&pi$d=$ S0103-11042014000600221>. Acesso em: 20 jun. 2018.

GUIMARÃES, A. S. J. T. S.; NERY, A. B. A. V. A. A. O enfermeiro no contexto do Programa Nacional de Melhoria do Acesso e da Qualidade da Atenção Básica (PMAQ): relato de experiência. Revista Saúde e Com., v. 11, n. 2, p. 193200, 2015. Disponível em: <www.uesb.br/revista/rsc/v11/ v11n2a010.pdf>. Acesso em: 20 fev. 2018.

JUNIOR, H. M. M.; PINTO, H. A. Atenção Básica enquanto ordenadora da rede e coordenadora do cuidado: ainda uma utopia? Divulgação em Saúde Para Debate, Rio de Janeiro, n. 51, p. 14-29, out. 2014. Disponível em: <cebes.org. br/site/wp-content/uploads/2014/12/Divulgacao-51.pdf>. Acesso em: 20 jun. 2018.

LIMÃO, N. P. et al. Equipamentos e insumos odontológicos e sua relação com as unidades da atenção primária à saúde. Revista Brasileira de Promoção da Saúde, Fortaleza, v. 29, n. 1, p. 84-92, jan./mar. 2016. Disponível em: <periodicos. unifor.br/RBPS/article/view/4035/pdf>. Acesso em: 20 jun. 2018.
LINHARES, P. H. A.; LIRA, G. V.; ALBUQUERQUE, I. M. A. N. Avaliação do Programa Nacional de Melhoria do Acesso e da Qualidade da Atenção Básica no estado do Ceará. Revista Saúde em Debate, Rio de Janeiro, p. 195-208, out. 2014. Disponível em: <www.scielo.br/pdf/sdeb/v38nspe/ 0103-1104-sdeb-38-spe-0195.pdf>. Acesso em: $17 \mathrm{dez}$. 2018.

MACHADO, M. F. A. S. et al. Programa saúde na escola: estratégia promotora de saúde na atenção básica no Brasil. J. Hum. Growth, São Paulo, v. 25, n. 3, 2015. Disponível em: <pepsic.bvsalud.org/scielo.php?pi$\mathrm{d}=$ S0104-12822015000300009\&script=sci_arttext\&tln$\mathrm{g}=\mathrm{pt}>$. Acesso em: 20 jun. 2018.

MEDRAdO, J. R. S.; CASANOVA, A. O.; OlIVEIRA, C. C. M. Estudo avaliativo do processo de trabalho nas equipes de atenção básica a partir do PMAQ-AB. Revista Saúde Debate, Rio de Janeiro, v. 39, n. 107, p. 976-978, out./dez. 2015. Disponível em: <WWW.scielo.br/pdf/sdeb/v39n107/ 0103-1104-sdeb-39-107-00984.pdf>. Acesso em: 20 jun. 2018.

MENDES, K. D. S.; SILVEIRA, R. C. C. P.; GALVÃO, C. M. Revisão integrativa: método de pesquisa para a incorporação de evidências na saúde e na enfermagem. Texto completo. Enfermagem, Florianópolis, 17(4), p. 758-64, out./dez. 2008.

MICLOS, P. V.; CALVO, M. C. M.; COLUSSI, C. F. Avaliação do desempenho da Atenção Básica nos municípios brasileiros com indicador sintético. Revista Saúde Debate, Rio de Janeiro, v. 29, n. 107, p. 984-996, out./dez. 2015. Disponível em: <www.scielo.br/pdf/sdeb/v39n107/0103-1104-sdeb-39-107-00984.pdf>.

MOHER, D. et al. Reprint-preferred reporting items for systematic reviews and meta-analyses: the PRISMA statement. PhysicalTherapy, Alexandria, v. 89, n. 9, p. 873-880, 2009.

MOREIRA, K. S.; VIEIRA, M. A.; COSTA, S. M. Qualidade da Atenção Básica: avaliação das Equipes de Saúde da Família. Revista Saúde \& Debate, Rio de Janeiro, v. 40, n. 111, p. 117-127, out./dez. 2016. Disponível em: <www.scielo. br/pdf/sdeb/v40n111/0103-1104-sdeb-40-111-0117.pdf>. Acesso em: 20 fev. 2018.

MOTA, R. R. A.; DAVID, H. M. S. L. Programa Nacional de Melhoria do Acesso e da Qualidade da Atenção Básica: questões a problematizar. Revista de Enfermagem da UERJ, Rio de Janeiro, v. 23, n. 1, p. 122-127, jan./fev. 2014. Disponível em: <www.facenf.uerj.br/v23n1/v23n1a20.pdf>. Acesso em: 20 jun. 2018.

PIMENTEL, F. C. et al. Caracterização do processo de trabaIho das equipes de saúde bucal em municípios de Pernambuco, Brasil, segundo porte populacional: da articulação comunitária à organização do atendimento clínico. Cadernos de Saúde Pública, Rio de Janeiro, v. 28, supl., 2012. Disponível em: <www.scielo.br/pdf/csp/v28s0/15.pdf>. Acesso em: 17 dez. 2018.

PINTO, H. A.; SOUSA, A. N. A.; FLORÊNCIO, A. A. O Programa Nacional de Melhoria do Acesso e da Qualidade da Atenção Básica: várias faces de uma política inovadora. Revista Saúde em Debate, Rio de Janeiro. v. 38, p. 358-372, 
2012. Disponível em: <www.scielo.br/pdf/sdeb/v38nspe/ 0103-1104-sdeb-38-spe-0358.pdf>. Acesso em: $17 \mathrm{dez}$. 2018.

PINTO, H. A. et al. Atenção Básica e Educação Permanente em Saúde: cenário apontado pelo Programa Nacional de Melhoria do Acesso e da Qualidade da Atenção Básica (PMAQ-AB). Revista Divulgação em Saúde para Debate, Rio de Janeiro, n. 51, p. 145-160, out. 2014. Disponível em: <cebes.org.br/site/wp-content/uploads/2014/12/Divulgacao-51.pdf>. Acesso em: 20 jun. 2018.

PINTO, H. A.; SOUSA, A. N. A.; FERLA, A. A. O Programa Nacional de Melhoria do Acesso e da Qualidade da Atenção Básica: várias faces de uma política inovadora. Revista Saúde em Debate, Rio de Janeiro, v. 38, out. 2014. Disponível em: <www.scielo.br/scielo.php?script=sci_arttext_pr\&pi$\mathrm{d}=$ S0103-11042014000600358\&tlng=pt>. Acesso em: 20 jun. 2018.

PROTASIO, A. P. L. et al. Satisfação do usuário da Atenção Básica em Saúde por regiões do Brasil: 10 ciclo de avaliação externa do PMAQ-AB. Revista Ciência \& Saúde Coletiva, v. 22, n. 6, jun. 2017. Disponível em: <https:// www.scielosp.org/scielo.php?script=sci_arttext\&pi$\mathrm{d}=$ S1413-81232017000601829>. Acesso em: 20 jun. 2018.

RIBEIRO, J. P. et al. Acessibilidade aos serviços de saúde na Atenção Básica do Estado de Goiás. Revista Eletrônica de Enfermagem, v. 17, n. 3, jul./set. 2015. Disponível em: <https://www.fen.ufg.br/revista/v17/n3/pdf/v17n3a04.pdf>. Acesso em: 20 jun. 2018.

SEIDL, H. et al. Gestão do trabalho na Atenção Básica em Saúde: uma análise a partir da perspectiva das equipes participantes do PMAQ-AB. Revista Saúde em
Debate, Rio de Janeiro, v. 38, out. 2014. Disponível em: <www.scielo.br/scielo.php?script=sci_arttext\&pi$d=$ S0103-11042014000600094>. Acesso em: 20 jun. 2018.

SOBRINHO, J. E. L. et al. Acesso e qualidade: avaliação das Equipes de Saúde Bucal participantes do PMAQ-AB 2012 em Pernambuco. Revista Saúde em Debate, Rio de Janeiro. v. 39, n. 104, p. 136-146, jan./mar. 2015. Disponível em: <https://www.scielosp.org/pdf/sdeb/v39n104/0103-1104-sdeb-39-104-00136.pdf>. Acesso em: 20 jun. 2018.

SOSSAI, T. A. et al. Evidências sobre o Programa Nacional de Melhoria do Acesso e da Qualidade da Atenção Básica. Revista Brasileira de Pesquisa e Saúde, Vitória, v. 18, n. 1, p. 111-119, jan./mar. 2016. Disponível em: <http://periodicos.ufes.br/RBPS/article/view/15142>. Acesso em: 20 fev. 2018.

SOUZA, M. F. et al. Care coordination in PMAQ-AB: an item response theory- based analysis. Rev. Saúde Pública, São Paulo, v. 51, 2017. Disponível em: http: //dx.doi. org/10.11606/s1518-8787.2017051007024>. Acesso em: 19 jul. 2018.

TEIXEIRA, M. B. et al. Avaliação das práticas de promoção da saúde: um olhar das equipes participantes do Programa Nacional de Melhoria do Acesso e da Qualidade da Atenção Básica. Revista Saúde em Debate, Rio de Janeiro, v. 38, out. 2014. Disponível em: <www.scielo.br/scielo.php?script=sci_arttext\&pid=S0103-11042014000600052>. Acesso em: 20 jun. 2018.

WHITTEMORE, R.; KNAFL, K. The integrative review: updated methodology. J AdvNurs., v. 52, n. 5, p. 546-53, 2005. 